[Aus dem Hygienischen Institut der Universität Berlin.]

(Direktor: Geheimrat Prof. C. Flügge.)-

\title{
Über die Bedeutung der Milch für die Verbreitung der Tuberkulose.
}

Von

Stabsarzt Dr. Köhlisch, früher kommandiert zum Institut.

Wenn Koch im Jahre 1901 in London die von den Erregern der Perlsucht dem Menschen drohende Gefahr als unerheblich bezeichnete, so wissen wir heute, daß der Mensch durch den Typus bovinus doch in nicht unerheblichem Umfang infiziert werden kann, und daß auch tödliche Tuberkulosen durch ihn verursacht werden.

Die Zusammenstellung von Kossel zählt 1602 Fälle menschlicher Tuberkulosen auf, die auf die Anwesenheit des Typus bovinus untersucht worden sind.

Dieser fand sich allein 126 mal, mit Humanus zusammen 9 mal. Zum weitaus größten Teil betreffen die Infektionen Kinder.

Bei ihnen ist vorzugsweise der Verdauungskanal - im weitesten Sinne - befallen. Man findet in der Zusammenstellung den Typus bovinus bei Erkrankungen der Halsdriisen in 40 Prozent, der Mesenterialdrüsen in 40 bis 50 Prozent der untersuchten Fälle, bei generalisierter Tuberkulose und Meningitis in geringerer Zahl.

Orth schätzt aus verschiedenen Statistiken die Häufigkeit der durch Typus bovinus verursachten primären intestinalen Tuberkulosen der Kinder auf 10 Prozent aller Tuberkulosen der Kinder überhaupt

Hierzu kommt, daß nach Untersuchungen von Behring, Orth, Römer, Bacmeister diese Infektionen im Kindesalter eine relative Dis- 
position der Lungen für die Phthise des Erwachsenen schaffen, wodurch die Bedeutung des Typus bovinus noch erhöht wird.

Die Infektion gerade des Verdauungskanals der Kinder durch Typus bovinus läßt zunächst an die Kuhmilch als Ursache denken. Dementsprechend zeigen Tabellen von Fraser, daß bei Brustkindern der Typus humanus kaum, bei Flaschenkindern fast ausschließlich gefunden wird, und daß, je jünger das Kind ist, um so häufiger Typus bovinus gefunden wird.

Daß die Kuhmilch Tuberkelbazillen enthält, wissen wir aus zahlreichen Untersuchungen. Sie sind nachgewiesen worden nicht nur in der Milch eutertuberkulöser Kühe, sondern anscheinend auch in der eutergesunder, aber sonst irgendwie tuberkulöser Kühe. Allerdings ist Joest der Ansicht, daß man bei mikroskopischer Durchmusterung der Euter doch immer einen kleinen tuberkulösen Herd im Euter findet.

Mit der Feststellung eines gelegentlichen Tuberkelbazillengehalts der Kuhmilch ist aber erst die Möglichkeit einer Infektion durch sie gegeben. Der Umfang und die Bedeutung dieser Infektiosität hängt ab von den Bazillenmengen, die nötig sind, um vom Darm aus eine Infektion herbeizuführén, ụnd von den Bazillenmengen, die man demgegenüber in der Milch findet.

Die Versuche von Reichenbach und von Findel zeigen, daß bei fortgesetzter Fütterung einige hundert, bei einmaliger immerhin viele Millionen Tuberkelbazillen nötig sind, um Versuchstiere vom Darm aus zu infizieren. Ob die Verhältnisse für den Menschen ebenso liegen, wissen wir nicht. Auch weisen Rogozinski, Fold, Selter u. a. darauf hin, daß der verletzte Darm viel leichter Bakterien durchläßt, was um so bedeutungsvoller ist, als ein menschlicher Darm mit tadellosem Epithel eine Seltenheit ist. Und Behring lehrt, daß der Darm Neugeborener durchlässiger ist, als der Erwachsener.

Immerhin scheint es, als ob ziemlich große Mengen Tuberkelbazillen in der Milch enthaiten sein müßten, um eine Infektion vom Darm aus zu bewirken.

Nun fanden Ostertag und Bongert allerdings, daß die Milch eutertuberkulöser Kühe noch in Verdünnungen von 1:1 Million, ja sogar 1:1 Billion für Meerschweinchen bei subkutaner und intraperitonealer Impfung infektiös sei.

Bei Ostermanns gleichen Versuchen war die Grenze der Infektiosität der Milch bei der Verdünnung 1:50000 noch nicht erreicht. Er schätzt danach, daß die Marktmilch durch eine eutertuberkulöse Kuh so infiziert werden kann, daß sie 1000 Tuberkelbazillen in $1 \mathrm{ccm}$ enthält. 
So scheint es also, als ob recht wohl eine wirksame Menge Tuberkelbazillen auch in der Marktmilch, die ja aus mehreren Herden, jedenfalls von vielen Kühen gemischt ist, vorhanden sein kann.

Tatsächliche Feststellungen darüber lagen aber noch nicht vor, und so wurde ich von Herrn Geheimrat Flügge zu einigen darauf bezüglichen neuen Untersuchungen angeregt.

Ich habe gewöhnliche Milch, die ich aus den verschiedensten Verkaufsstellen Breslaus entnahm, Meerschweinchen intraperitoneal verimpft. In jedem Versuch wurden 2 Tiere mit unverdünnter Milch, je eins mit den Verdïnnungen $1: 10,1: 100,1: 1000$ geimpft, im ganzen also bei jedem Versuch 5 Tiere. Das injizierte Flüssigkeitsquantum betrug jedesmal $1 \mathrm{ccm}$.

Ich tötete die Tiere der ersten Versuche 2 bis 3 Monate nach der Injektion. Als ich jedoch bei Fränkel und Baumann fand, daß Krankheitszeichen bei Infektion mit ganz geringen Mengen Tuberkelbazillen oft erst nach 7 bis 9 Monaten auftreten können, ließ ich die anscheinend gesunden Tiere, deren Injektionsstelle und deren Drüsen ich öfters befühlte, bis zu 7 Monaten leben und tötete zwischendurch nur solche, die offenbar krank waren.

Betrachten wir die Versuche insgesamt, so sehen wir, daß von den 23 Versuchen, die in der Tabelle zusammengestelllt sind, 12 (=52.2 Proz.) ein positives Resultat ergaben. Bei 8 Versuchen führte im wesentlichen lediglich die Impfung mit unverdïnnter Milch zu einer Erkrankung der Tiere; nur bei einem Versuch.(2) enthielten alle う́ Verdünnungen Tuberkelbazillen; hier ist es also fraglich, ob die Grenze der Infektiosität dieser Milchprobe schon erreicht war.

Auffallend sind die 3 Versuche 8, 15 und 22, in denen ausschließlich die mit den Verdünnungen 1:100 oder 1:1000 geimpften Tiere erkrankten. Indessen zeigen auch die Versuche 3 und 4 Luicken in der Reihe der Verdünnungen. Es beweist dies nur, daß bereits unter natürlichen Verhältnissen durch die Mischung mit anderer gesunder Milch die Verdünnung der infizierten Milch so weit getrieben ist, daß nicht jeder Kubikzentimeter Tuberkelbazillen enthält. Die Verteilung der festen, Tuberkelbazillen enthaltenden Partikel in der Milch ist offenbar sehr ungleichmäßig, und dementsprechend wird in den Versuchen mit unverdünnter, wie mit verdünnter Milch gelegentlich und rein zufällig einmal ein positives Resultat. erzielt.

Systematisch sind Versuche über die Erfolge extremer Verdünnungen von Fränkel und Baumann angestellt.

Sie infizierten Kaninchen, weiße Mäuse und schließlich besonders Meerschweinchen mit Aufschwemmungen von Tuberkelbazillen in sehr zahlreichen Verdünnungen. Bis zu einer bestimmten Grenze erkrankten 
Bedeutung der Milch für die Verbreitung der Tuberkulose. 199

Die Anzahl Tage unter den +-oder - Zeichen bedeuten die Zeit, die das Versuchstier nach der Injektion noch gelebt hat.

I. Breslauer Molkereien.

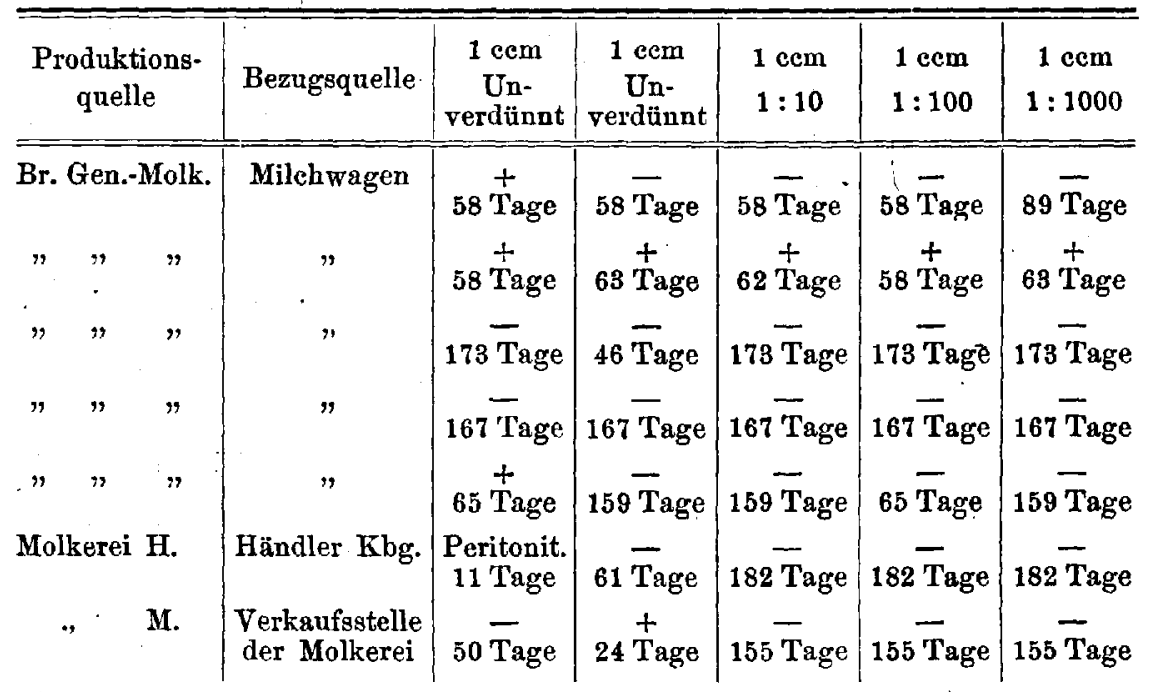

II. Dominial-Molkereien.

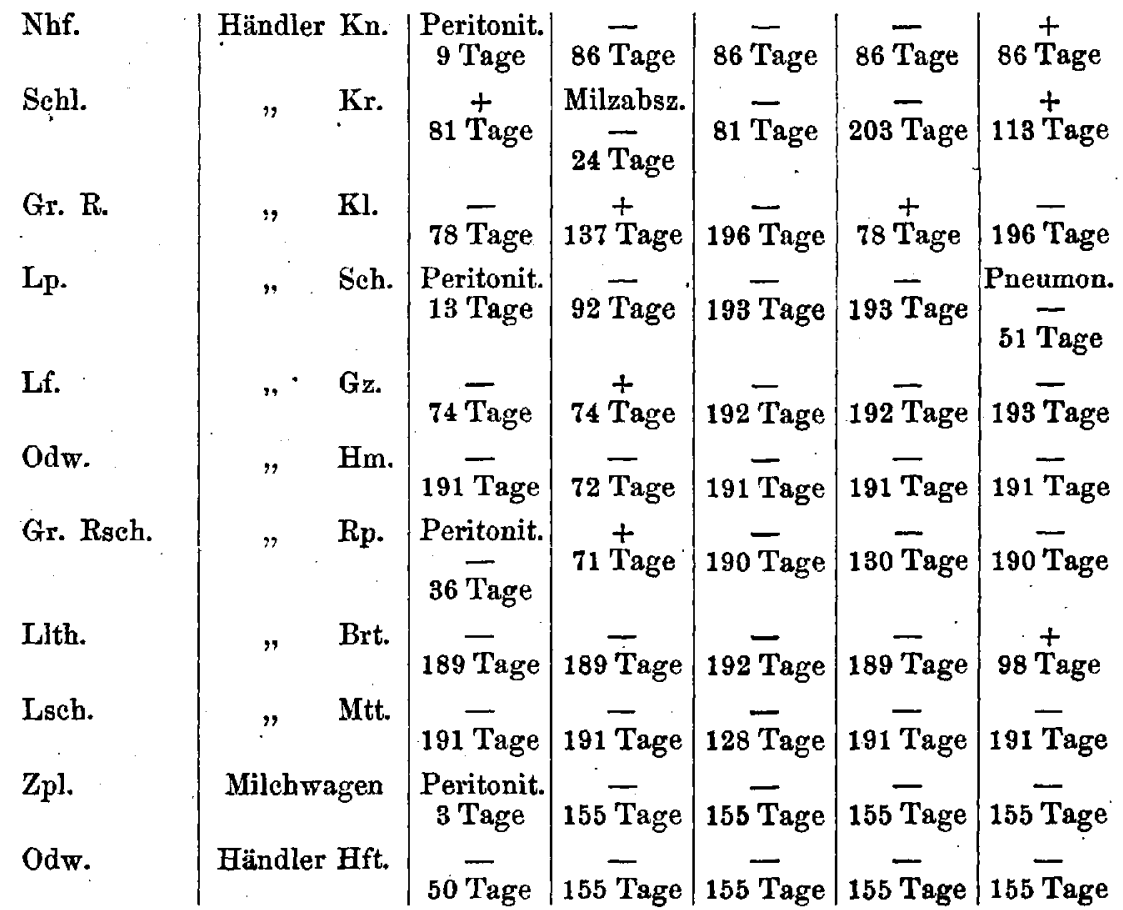


III. Bauern-Milch:

\begin{tabular}{|c|c|c|c|c|c|c|}
\hline $\begin{array}{c}\text { Produktions- } \\
\text { quelle }\end{array}$ & Bezugsquelle & $\begin{array}{c}1 \text { cem } \\
\text { Un- } \\
\text { verdünnt }\end{array}$ & $\begin{array}{c}1 \mathrm{~cm} \\
\text { Un- } \\
\text { verdünnt }\end{array}$ & $\begin{array}{l}1 \mathrm{ccm} \\
1: 10\end{array}$ & $\begin{array}{l}1 \mathrm{cem} \\
1: 100\end{array}$ & $\begin{array}{c}1 \mathrm{cem} \\
1: 1000\end{array}$ \\
\hline Bauern Schw. & Händler Beg. & $153^{+}$Tage & 162 Tage & 162 Tage & 162 Tage & 162 Tage \\
\hline$"$ & Milchwagen & $\begin{array}{c}\text { Peritonit. } \\
\text { 3 Tage }\end{array}$ & $\begin{array}{l}\text { Peritonit. } \\
12 \text { Tage }\end{array}$ & $\begin{array}{c}\text { Peritonit. } \\
5 \text { Tage }\end{array}$ & $155 \overline{\text { Tage }}$ & $15 \overline{\text { Tage }}$ \\
\hline$\eta \quad$ P. Ndf. & Händler Schm. & $15 \overline{\text { Tage }}$ & 155 Tage & 155 Tage & $\left\{\begin{array}{c}\text { Urs. unbek. } \\
38 \text { Tage }\end{array}\right.$ & ${ }_{155} \overline{\text { Tage }}$ \\
\hline $\begin{array}{l}5 \text { Bauern, die } \\
\text { der Molkerei } H \text {. } \\
\text { liefern }\end{array}$ & $\begin{array}{l}\text { direkt aus der } \\
\text { Molkerei }\end{array}$ & $50 \overline{\text { Tage }}$ & $\begin{array}{c}\text { Peritonit. } \\
6 \text { Tage }\end{array}$ & $50 \overline{\mathrm{Tage}}$ & 50 $\stackrel{+}{\text { Tage }}$ & ${ }_{155 \text { Tage }}$ \\
\hline $\begin{array}{c}\text { Einige Bauern, } \\
\text { die der Molkerei } \\
\text { M. liefern }\end{array}$ & $\begin{array}{l}\text { direkt aus der } \\
\text { Molkerei }\end{array}$ & $\left|\begin{array}{c}\text { Preumon. } \\
41 \overline{\text { Tage }}\end{array}\right|$ & $149 \overline{\mathrm{T}}$ age & $\begin{array}{l}\text { Urs. unbek. } \\
50 \text { Tage }\end{array}$ & 149 Tage & 78 Tage \\
\hline
\end{tabular}

die Tiere jeden Verdünnungsgrades; dann immer nur einzelne in der Reihe der Verdünnungen, während andere übersprungen wurden.

Bei Plattenkulturen mit Verdünnungen einer Prodigiosusaufschwemmung erhielten sie ganz analoge Resultate.

Die theoretische Erwägung läßt das ja auch nur natürlich erscheinen.

Es war nun weiter von Interesse, festzustellen, in welchem Maße die einzelnen Gruppen von Proben verschiedener Herkunft an den positiven Resultaten beteiligt sind.

Ich habe daher 1. die Milch verschiedener großer Breslauer Molkereien, 2. die Milch von Dominial-(Guts-) Molkereien, die zum Teil nicht nur die Milch der Dominialherden, sondern auch aus Bauernwirtschaften verarbeiteten, und 3. Milch auschließlich aus Bauernwirtschaften in der Tabelle voneinander getrennt.

Einen wesentlichen Unterschied zwischen den verschiedenen Milchsorten fand ich nicht. Von 11 Versuchen mit Dominialmileh waren $6(=55$ Prozent) positiv, von 7 Versuchen aus den Großstadtmolkereien 4 (= 57 Prozent), von 5 Vèrsuchen mit Bauernmilch 2 (= 40 Prozent). Das beste Resultat hat also noch die Bauernmilch gegeben, wodurch man, falls die kleinen Zahlen allgemeine Gültigkeit haben, an die Angabe von Behrings erinnert wird, daß die Zahl der tuberkulösen Kühe mit der Größe der Herde nicht nur relativ, sondern vielmehr absolut wächst.

Auch Eber fand übrigens - aber in viel deutlicheren Untersehieden - daß Bauern- und Kleinstadtmilch viel seltener Tuberkelbazillen enthält, als Grọstadtmilch. 
Die Milchprobe (2), die in allen Verdünnungen bis $1: 1000$ positive Resultate ergab, entstammte auffallenderweise unserer größten Molkerei.

Wir dürfen also als Ergebnis meiner Untersuchungen verzeichnen; $\mathrm{daB}$ sich gelegentlich große Mengen Tuberkelbazillen in der Marktmilch finden. Daß damit die Möglichkeit einer Infektion durch Milch immer einmal gegeben sein kann, beweist namentlich der Versuch 2, bei dem mit allen Verdünnungen bis 1:1000 ein positives Resultat erzielt wurde. Nehmen wir selbst nur 1000 Tuberkelbazillen pro Kubikzentimeter an, eine Zahl, die ja der Ostermannschen Schätzung entspricht, so haben wir in $1 / 2$ Liter bereits 500000 Tuberkelbazillen und kommen damit der Dosis, die bei Reichenbach in 51 maliger Fütterung bei 2 Tieren Tuberkulose erzeugte, schon recht nahe. Tatsächlich haben wir aber offenbar mit der Verdünnung 1:1000 die Grenze der sicheren Infektiosität der Milch noch nicht erreicht. Zwar ist bei einmaligem Genuß eine Infektion noch nicht mit Notwendigkeit $\mathrm{zu}$ erwarten, aber bei mehrmaligem Genuß ist sie doch schon .wahrscheinlich.

Allerdings ist das bei 26 Versuchen der einzige Fall, und der Ausfall der übrigen Versuche spricht nicht gerade für eine größere Bedeutung der Milch bei dem Zustandekommen der menschlichen Tuberkulose. In fast allen positiven Versuchen ist schon mit $1 \mathrm{ccm}$ unverdünnter Milch die Grenze erreicht, und die erzielten Infektionen sind nur ,Zufallstreffer". Ich glaube, daß wir bei diesen 11 Versuchen kaum mehr als 10 Tuberkelbazillen im Kubikzentimeter unverdünnter Milch annehmen dürfen. Das macht je nach dem Alter des Kindes für die Tagesportion von $1 / 2$ bis 1 Liter 5000 bis 10000 Tuberkelbazillen, und diese Menge wird vermutlich auch bei fortgesetztem Rohgenub der Milch bei gesundem Darm eine Infektion nicht bewirken.

Berücksichtigt man andererseits, daß der Darm Neugeborener für Tuberkelbazillen durchlässiger ist, und daß der menschliche Darm überhaupt selten frei von Epitheldefekten ist, so resultiert trotzdem eine gewisse, wenn auch nicht verbreitete Infektionsmöglichkeit.

Mit diesen Ergebnissen stimmen auch die in den Statistiken berechneten Zahlen der intestinalen Tuberkulosen der Kinder und der bovinen Infektionen überein. Aus beiden Untersuchungen dürfen wir folgern, daß der Milch eine wesentliche Rolle bei der Entstehung und Verbreitung der Tuberkulose nicht zukommt. Es kommt hinzu, daß eine Infektionsgefahr nur dann von der Milch ausgeht, wenn das Kochen der Säuglingsmilch unterlassen ist, und dab eine so grobe Nachlässigkeit doch immerhin selten ist. Aber immerhin bleibt ein Rest von Gefahr durch den Typus bovinus bestehen, und dieser rechtfertigt durchaus 
die strengen Maßnahmen, die im Kampfe gegen die Tuberkulose für tuberkelbazillenfreie Marktmilch empfohlen und zum Teil schon durchgeführt sind.

\section{Literatur.}

Die im Text angeführten Arbeiten sind in den ausführlichen Literaturverzeichnissen bei den Abhandlungen von

Römer, Die Ansteckungswege der Tuberkulose im Handbuch der Tuberkulose, und von

Bacmeister, Die Entstehung der Langenphthise. Berlin, Springer, 1914, zu finden. 